\title{
Fuzzy approach to improving route stability of the AODV routing protocol
}

\author{
Nihad I. Abbas ${ }^{1 *}\left(\mathbb{D}\right.$, Mustafa Ilkan ${ }^{2}$ and Emre Ozen ${ }^{2}$
}

\begin{abstract}
A mobile ad hoc network (MANET) is a group of autonomous mobile nodes that wirelessly communicate with each other to form a wireless dynamic topology network. It works without requiring any centralized pre-existing administration units (infrastructure less network). There are many studies that focus on improving source-destination route stability and lifetime by modifying the existing MANET routing protocols. In this paper, a fuzzy-based approach is proposed to enhance the ad hoc on-demand distance vector (AODV) reactive routing protocol's performance by selecting the most trusted nodes to construct the route between the source and destination nodes. In this scheme, the nodes' parameters, such as residual energy, node mobility, and number of hop counts, are fed through a fuzzy inference system to compute the value of the node trust level, which can be used as a metric to construct an optimal path from source to destination. The results of the simulation show that the proposed approach performs better than the traditional AODV routing protocol and minimum battery cost routing protocol in terms of average control overhead, packet delivery ratio, network throughput, and average end-to-end delay
\end{abstract}

Keywords: AODV, Reactive routing protocol, MANET, Fuzzy logic, Trust nodes, MBCR protocol

\section{Introduction}

Mobile ad hoc networks (MANETs) have received considerable attention over the past few decades. The rapid deployment of wireless MANETs in many emergency cases such as disaster areas, rescue operations, and battlefield operations make these types of networks more attractive than other solutions. A MANET network is a collection of mobile nodes that temporarily communicate to form a special sort of wireless network. The nodes in the MANET organize and configure themselves dynamically without the need of an administrator. Each node in the MANET can join or leave the network arbitrarily and is free to move at any speed and in any direction independently. Battery powered devices, such laptops, PDAs, or smartphones, are widely used in MANETs as mobile nodes. The limited energy resources of these devices force the wireless MANET developers to adapt a multi-hop route communication strategy in order to preserve the node's energy and prolong the MANETs lifetime [1]. Unfortunately, route failures frequently occur in MANETs

\footnotetext{
* Correspondence: nehadiabbas@yahoo.com

${ }^{1}$ Department of Computer Engineering, Eastern Mediterranean University,

Famagusta, N. Cyprus

Full list of author information is available at the end of the article
}

because of the mobile nodes' mobility and limited energy resources. For this reason, therefore, an efficient routing protocol is needed to reconnect the source-destination route whenever routes are broken, and the routing protocol algorithms must react rapidly to environmental changes.

Many simple MANET-reactive routing protocols use a single metric like the shortest path (SP), signal strength, or node battery's residual to construct the route for data transmission. This single-metric route selection is not sufficient to construct a stable route because it may cause frequent route failures that stimulate the routing protocol algorithms to rediscover a new route each time a route is broken. The operations of route discovery consume extra network resources, degrading network performance, minimizing network lifetime, and leading to network partitioning problems. In contrast, improving the efficiency of the route selection scheme in a MANET can be achieved by combining multiple routing metrics using an adaptive intelligent tool to choose the most trustworthy nodes from which the best route to a destination can be constructed $[2,3]$.

The ad hoc on-demand distance vector (AODV) routing protocol is one of the most popular reactive routing 
protocols in wireless MANETs. It uses the minimum hop-count criteria (SP) to select the route for data transmission without taking into account a path's link stability factors or node quality when constructing the route. A node in a MANET running the AODV protocol must flood routing control packets over the network each time it needs to discover a route to a destination. Such nodes are likely to exhaust their energy resources and deplete their battery power rapidly. Hence, node cooperation is needed to preserve MANET network resources and support the wireless network performance effectively [4]. This ideal cooperative environment, generally, is not achieved in traditional, simple MANET routing protocols. The behavior of a MANET node changes continuously over time, depending on the wireless network environment. However, a variety of concepts, schemes, and models have been proposed to achieve intelligent services and networks. Adding open programming and management abilities to the nodes can enhance the new network services. This feature of programmable network elements moves the control and management network system toward an adaptively evolutionary computing system with a variety of genetic algorithms and evolutionary programming [5]. In this work, a fuzzy inference system is proposed as an adaptive computational approach to compute a node's trust value and introduce an efficient routing scheme by selecting the most trustworthy nodes to establish a stable route. Using the concept of node trust when building stable routes decreases the probability of route breaks during the data relay period. This, consequently, minimizes the amount of unnecessary overhead control packets transmitted over the network in the route discovery stage. In addition, it preserves network resources and improves network performance. Finally, it is shown that the proposed intelligent fuzzy-based AODV-modified scheme performs better than the simple classical MANET routing protocols. The organization of the rest of this paper is as follows: Section 2 presents a literature review; Section 3 describes the proposed fuzzy-based algorithm used in this article; Sections 4 and 5 present the simulation environment and discuss the results, respectively; and Section 6 concludes the paper.

\section{Literature review}

Different routing protocols have been proposed for wireless MANETs. These protocols can be generally categorized into two types: table-driven (proactive) routing protocols, and on-demand (reactive) routing protocols $[6,7]$. In the proactive routing protocol algorithms, the routing table of each node in the network includes all possible routes to all destination nodes. It updates its routing information table periodically for any changes that occur in the network topology, irrespective of the route requirements, resulting in a waste of network resources. The proactive routing protocols exhibit less efficient performance than reactive routing protocols in high-mobility and high-density wireless network applications [8,9]. Reactive routing protocols have been proven to be more effective and have a better performance record in wireless MANETs. Various aspects of reactive routing algorithms of multi-hop wireless networks are the effects of network parameters and operation environments on route stability and MANET performance [10, 11].

Marwaha et al. [12] proposed an evolutionary ad hoc on-demand fuzzy routing algorithm to determine the best route to achieve various objective performances in wireless MANETs. A fuzzy cost evaluation function that combines different routing metrics such as remaining node battery, node queue length, and the signal strength between two intermediate nodes is used to select the route with the minimum fuzzy cost value. Their simulation results demonstrate the superiority of the proposed scheme over conventional MANET routing schemes.

Srivastava et al. [13] considered node mobility and proposed an adaptive algorithm for establishing a stable route for data transmission, regardless of the neighboring nodes' characteristics. They introduced a communication link expiration time in the evaluation of route stability. The proposed adaptive routing scheme improves the performance of MANET network effectiveness. A link failure problem often causes degradation of the network performance; hence, Hundal et al. [14] suggested a new method to reduce the effects of link failure in MANETs. They defined a signal strength parameter to determine a stable path for packet transmission. High-speed stable routes are required to ensure a better packet delivery ratio between network nodes. Hence, dynamic switching between nodes was introduced by the authors. They suggested a method to select the neighboring node with maximum signal strength for data transmission. The scheme was used to ensure a stable route path and reduce the hop count between the source and destination when compared with traditional techniques.

In AODV routing, a tradeoff strategy between an energy-aware routing algorithm and link stability was investigated by $\mathrm{Xu}$ et al. [15]. The purpose of their protocol was to establish a highly stable route using the information of node energy and link quality. In addition, the routing algorithm considers a tradeoff between the route stability and hop count to choose the best route with respect to high stability factor and low hop count. Their proposal includes energy awareness and link stability metrics in the routing design. They suggest a method to estimate the route's link lifetime by observing the relative node movement over a specific time period. Their proposed approach improves the network utilization considerably. 
An improved preemptive local repairing mechanism (PLRM) for the AODV protocol was proposed by Zhang et al. [16]. The authors suggested an approach to avoid route link breakage by observing the quality of route links and other performance-degrading factors. Their approach allows for the minimization of overhead control packets and repair of the route length of a process. They compared their proposed technique with other modified AODV route-repairing approaches. The proposed PLRM showed the best performance in terms of control overhead, delivery ratio, and packet delay.

A novel scheme to improve the AODV protocol by creating a cognitive process was described by Ghorbani et al. [17]. The route discoveries of their algorithm can reduce the failure rate of the links via a kind of intelligent delay of the route discovery schemes. A new parametric algorithm was introduced to change the AODV route discovery algorithm, and then, a network of learning automata was used to set its parameters. Nasiri et al. [18] defined a method to predict the lifetime of route links, depending on the information collected about node mobility and network environments. They demonstrated the impact of link reliabilities on the network performance by reducing the wait time and minimizing the control overhead signals in their comprehensive studies of network parameters.

Lim et al. [19] focused their efforts on trying to determine a more stable route by considering the route lifetime and link stability of different protocols such as stabilitybased signal-adaptive routing (SSA) and associativitybased routing (ABR). A comparison of this routing algorithm with a locally optimal algorithm showed that it improved the estimation model link stability and found the best routes with longer lifetimes.

Many stability-oriented routing algorithms focus on how to discover a suitable route for transferring packets through intermediate nodes, but little attention is given to discovering a stable route that floods only the minimum number of overhead control packets. In the past few years, several fuzzy-based protocols for MANETs have been proposed, forming a new field of research.

\section{Proposed fuzzy-based model}

This section describes the modification of the classical AODV protocol to improve the route selection scheme and enhance network performance by using a fuzzy logic inference system.

\subsection{Traditional AODV protocol overview}

AODV is one of the most popular wireless mobile reactive routing protocols used in the research environment. It supports multicast and unicast routing protocols. The source node starts a route discovery process whenever it has data packets to be sent. It floods a route request packet (RREQ) to all neighbor nodes in transmission range. Each node that receives the RREQ determines if it has a fresh route to the destination or is itself the destination, then replies back in unicast form a route replay packet (RREP) to the source node. The traditional AODV protocol uses the minimum hop-count (SP) parameter to select the route to the destination nodes, regardless of the nature of the nodes used to construct the route. However, if the source receives multiple RREP packets, the shortest hop-count route is selected. When a route link failure occurs, a route error packet (RERR) is created and passed back to the originator node. The source starts the process of route discovery process again if the route is still needed or more packets need to be sent [20].

In traditional AODV protocol, the source node floods a RREQ packet during the route discovery process stage. Any intermediate node that receives the RREQ packet rebroadcasts it (if the intermediate node is not a destination or does not have a fresh route to the destination) after incrementing the HOP-COUNT parameter by one. However, the intermediate node usually receives multiple RREQ packets of the same identification (ID) and sequence number with different HOP-COUNT values from its neighbors. Hence, the node examines each RREQ packet individually. If it has a lesser HOP-COUNT value than previously received RREQ packets with the same ID, then the node updates its reverse route table and rebroadcasts the RREQ packet. Otherwise, it discards the RREQ if it has a lower or equal sequence number. An intermediate node may propagate the same identification RREQ packet more than once, as illustrated in Fig. 1. Thus, the traditional AODV protocol MANETs unnecessarily consume energy and waste network bandwidth resources as well as increase network traffic, especially in high-density wireless MANETs [21].

\subsection{Proposed fuzzy AODV protocol}

In traditional AODV, the minimum number of hops metric is used to make a decision about route selection, but this is not a sufficient parameter for constructing the best route to a destination in a wireless MANET [12]. It does not consider other factors that may effect on the route quality, such as the received signal strength, node mobility, or node residual energy, among others. In the proposed fuzzy AODV, important node metrics such as node residual energy and mobility are considered to construct a reliable route and minimize the probability of route failure during data packet transmission. The choice of trustworthy nodes used to build a stable route in our fuzzy algorithm is based on the nodes that have a higher residual energy level and move with minimum speed. The proposed approach uses fuzzy logic techniques to determine a node's trust value by combining the residual energy and speed of each node in the MANET. The nodes with the highest trust values are selected to establish the best 


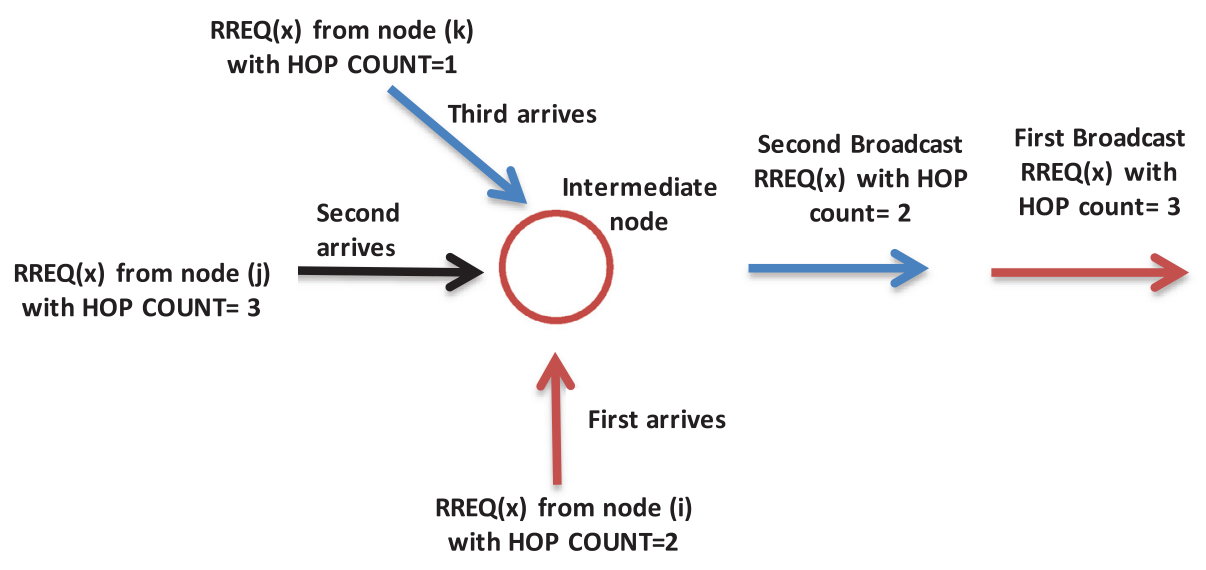

Fig. 1 Intermediate node RREQ broadcasting in classic AODV

route available to the destination node. Each intermediate node calculates its trust value whenever it receives the RREQ packet. The intermediate node initiates a timer if the RREQ packet has not been previously received. During the timer duration, the intermediate node receives more RREQ packets (of the same identification ID and sequence number) from its neighbors. The intermediate node selects the node with the best trust value (carried by the received RREQ packets) to update its reverse route table, which will be used to construct the reverse unicast route as a part of a reliable route establishment between source and destination. After the timer expires, the intermediate node forwards the RREQ, carrying the intermediate node's trust value to other neighbors, as shown in Fig. 2. The timer is used to examine the same RREQ packets that arrive at different times to the intermediate node, and then, the one with the highest trust value is forwarded.
This procedure to select the best path using trustworthy nodes minimizes the amount of overhead control packets flooded throughout the network and reduces the probability of network traffic congestion. A flow chart of the proposed algorithm is shown in Fig. 3.

\subsection{Fuzzy-based trust value computations}

Computational intelligence techniques have been extensively used in various fields of engineering research and control engineering and provide a very promising approach in computer communication routing algorithms [22-24]. Fuzzy logic theory was first proposed by Zadah in 1965 [25]. The basic fuzzy systems shown in Fig. 4 are suited for decision-making techniques. A fuzzy logic system describes the relationship between crisp inputs and output variables with the help of IF-THEN-based rules provided by the fuzzy system designer [26]. A fuzzy system consists of three

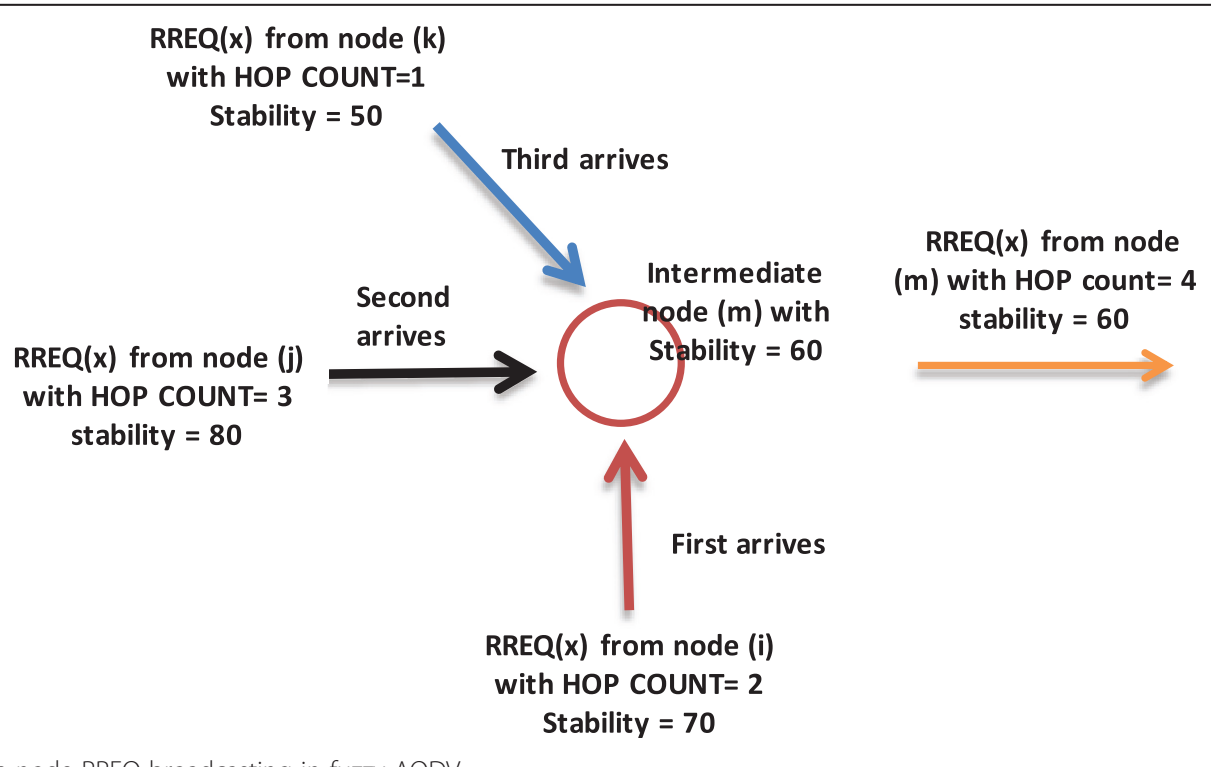

Fig. 2 Intermediate node RREQ broadcasting in fuzzy AODV 


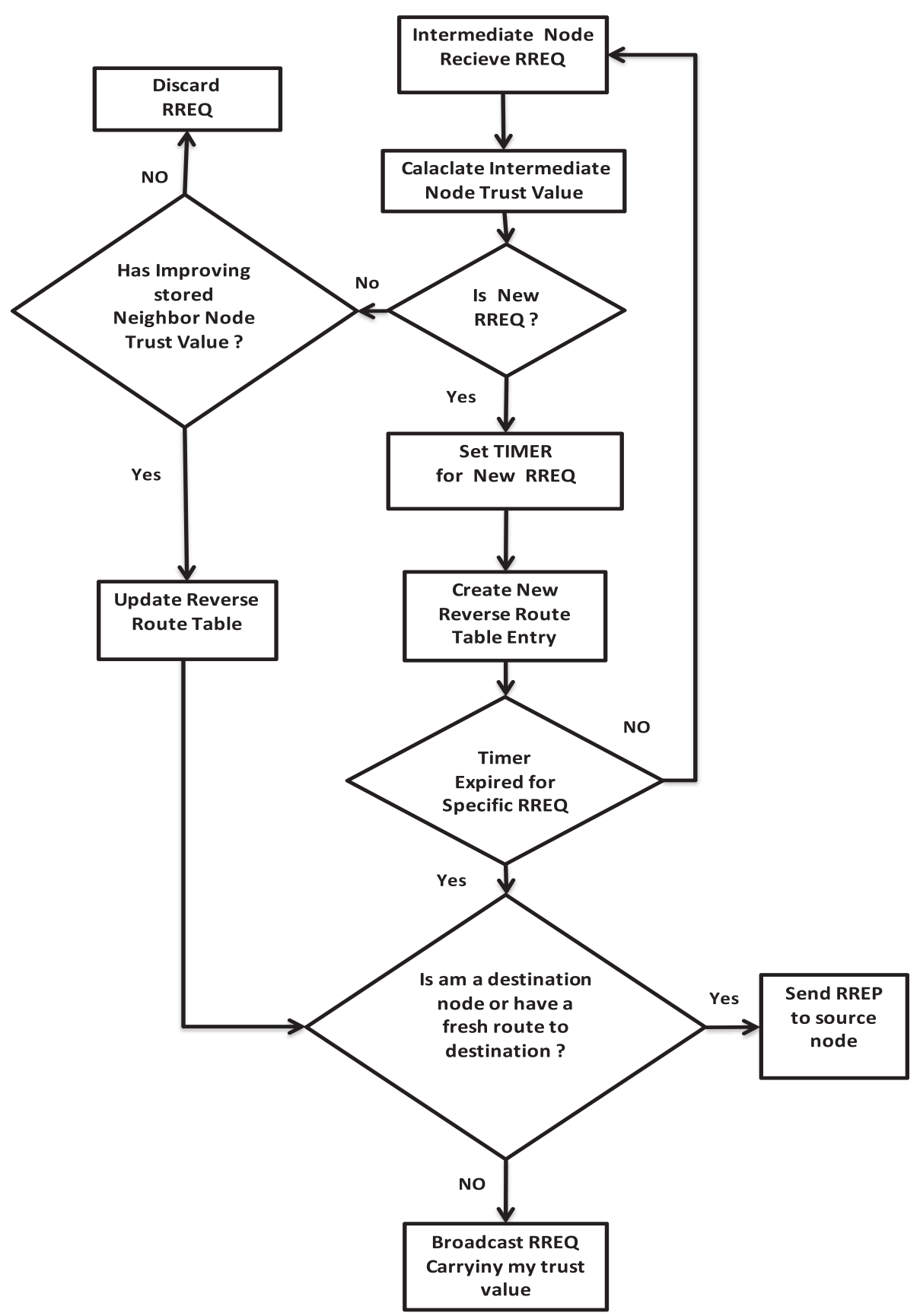

Fig. 3 Flowchart of the proposed fuzzy AODV algorithm

parts: fuzzification, defuzzification, and a fuzzy inference engine with IF-THEN-based rules. Fuzzification is responsible for representing decisive input variables in terms of fuzzy set membership functions, as shown in Fig. 5. Defuzzification converts the fuzzy output to decisive values using a mathematical formula, while the inference engine calculates the fuzzy output depending on the IF-THEN-based rules provided in Table 1.

Because of the correlation between the nodes' parameters, which have a range of values, the fuzzy logic system describes the effects of the different parameter interactions. Hence, to develop a fuzzy inference system, the input and output variables should be defined as membership functions. Fuzzy rules (IF-THEN) that connect the input memberships with the output membership are then suggested [27]. The membership function is a graphical interpretation of the input and output linguistic variables. The inputs in our case are node residual energy, speed, and hop-count value, and the output represent the node trust value (node quality). Triangular and trapezoid 


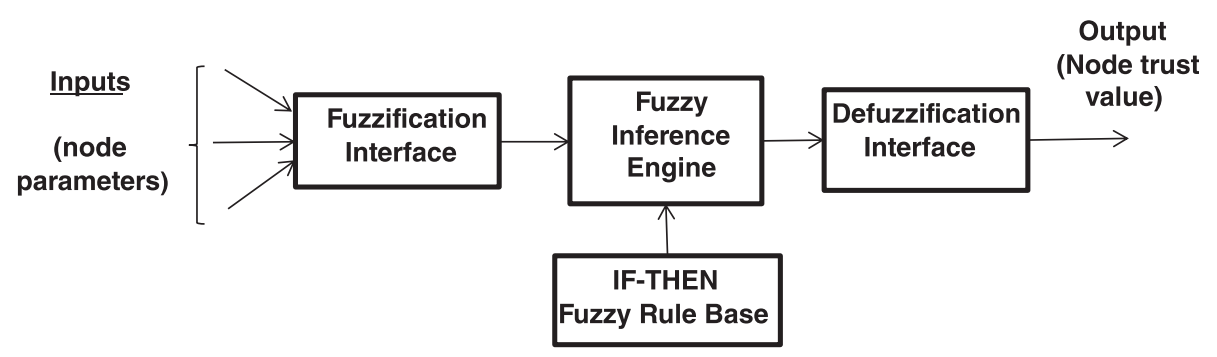

Fig. 4 Fuzzy logic system

membership functions described by Eqs. 1 and 2 below are used to describe the input and output membership degrees of the input and output variables for fuzzy inference. Node residual energy, which directly impacts the lifetime of a network, has an important influence on the node abilities of electromagnetic communication, packet transmission, reception, and internal computing processes [28]. For this reason, it is treated as a key input value in the fuzzy node trust value calculation. The input parameter of node speed also has a considerable effect on route stability; when the selected node moves rapidly out of communication range of the other participating route's nodes, the link is broken. Hence, nodes with the highest speed increase the probability of a route being broken and increase the overhead of control packet retransmission for route discovery. The third parameter of a fuzzy input variable is the number of hop-count values included in the RREQ packet, which represents route length. Generally, the route with the minimum number of hops is the best route if all nodes participating in the established shortest route have maximum residual energy and low speed. Hence, the hop-count parameter has the least significant effect on the output node trust value.

$$
\mu_{\mathrm{A} 1}(x)=\left\{\begin{array}{lc}
\frac{x-\mathrm{a} 1}{\mathrm{~b} 1-\mathrm{a} 1} & x \leq \mathrm{a} 1 \\
\mathrm{c} 1-x & \mathrm{a} 1 \leq x \leq \mathrm{b} 1 \\
\frac{\mathrm{c} 1-\mathrm{b} 1}{0} & \mathrm{~b} 1 \leq x \leq \mathrm{c} 1 \\
0 & x \geq \mathrm{c} 1
\end{array}\right.
$$

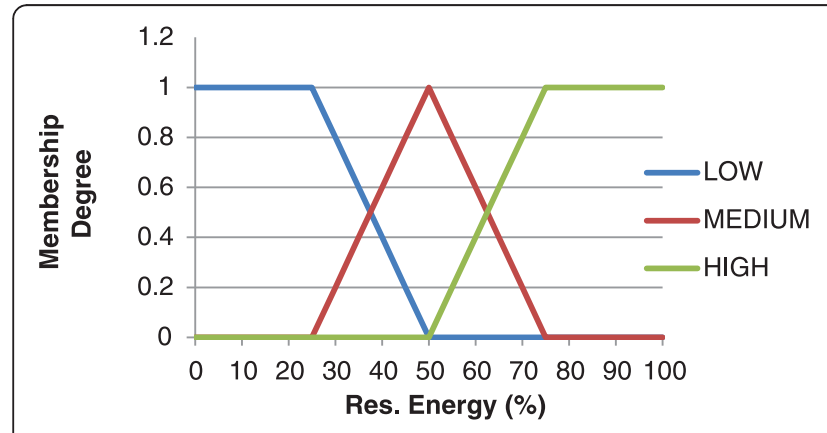

(a) Membership function of the residual energy input

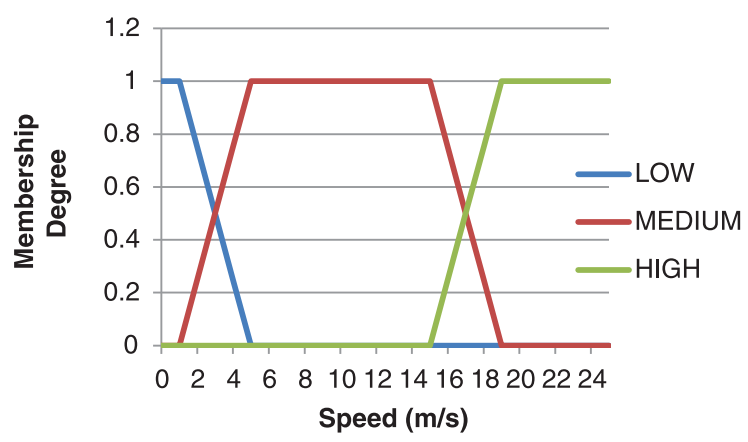

(b) Membership function of node speed input

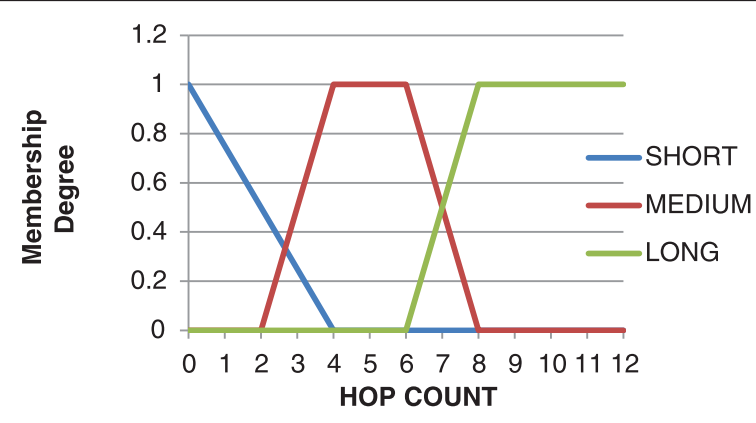

(c) Membership function of the HOP COUNT input

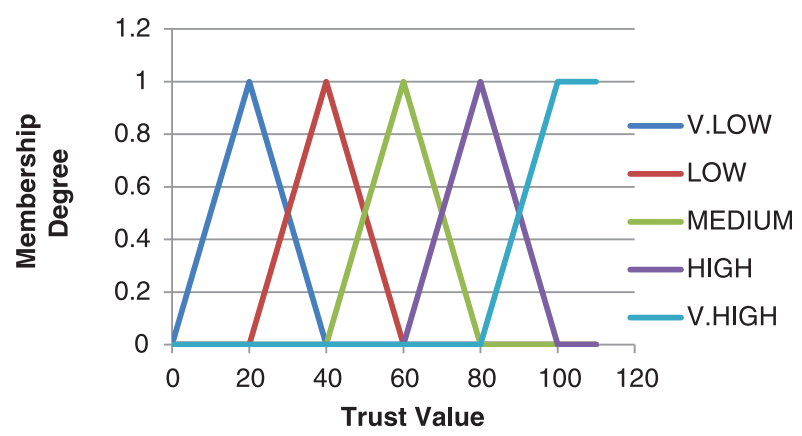

(d) Output membership function of node trust

Fig. 5 Fuzzy membership sets of the input and output variables. a Membership function of the residual energy input. b Membership function of node speed input. c Membership function of the hop-count input. $\mathbf{d}$ Output membership function of node trust 
Table 1 Fuzzy base rule set

\begin{tabular}{|c|c|c|c|c|c|c|c|c|c|c|c|}
\hline \multicolumn{3}{|l|}{ Inputs } & \multirow{2}{*}{$\begin{array}{l}\text { Output } \\
\text { Trust node }\end{array}$} & \multicolumn{3}{|l|}{ Inputs } & \multirow{2}{*}{$\begin{array}{l}\text { Output } \\
\text { Trust node }\end{array}$} & \multicolumn{3}{|l|}{ Inputs } & \multirow{2}{*}{$\begin{array}{l}\text { Output } \\
\text { Trust } \\
\text { node }\end{array}$} \\
\hline Res. energy & Node speed & Hop count & & Res. energy & Node speed & Hop count & & Res. energy & Node speed & $\begin{array}{l}\text { Hop } \\
\text { count }\end{array}$ & \\
\hline Low & Low & Short & Med & Med & Low & Short & High & High & Low & Short & V. High \\
\hline Low & Low & Med & Med & Med & Low & Med & High & High & Low & Med & V. High \\
\hline Low & Low & Long & Low & Med & Low & Long & High & High & Low & Long & V. High \\
\hline Low & Med & Short & Low & Med & Med & Short & Med. & High & Med & Short & High \\
\hline Low & Med & Med & Low & Med & Med & Med & Med. & High & Med & Med & High \\
\hline Low & Med & Long & V. Low & Med & Med & Long & Low & High & Med & Long & High \\
\hline Low & High & Short & Low & Med & High & Short & Med. & High & High & Short & High \\
\hline Low & High & Med & V. Low & Med & High & Med & Low & High & High & Med & Med \\
\hline Low & High & Long & V. Low & Med & High & Long & Low & High & High & Long & Med \\
\hline
\end{tabular}

$$
\mu_{\mathrm{A} 2}(x)=\left\{\begin{array}{cc}
0 & x \leq \mathrm{a} 2 \\
\frac{x-\mathrm{a} 2}{\mathrm{~b} 2-\mathrm{a} 2} & \mathrm{a} 2 \leq x \leq \mathrm{b} 2 \\
\frac{1}{\mathrm{~d} 2-x} & \mathrm{~b} 2 \leq x \leq \mathrm{c} 2 \\
\frac{\mathrm{d} 2-\mathrm{c} 2}{0} & \mathrm{c} 2 \leq x \leq \mathrm{d} 2 \\
0 & x \geq \mathrm{d} 2
\end{array}\right.
$$

\subsection{Fuzzy IF-THEN-based rules}

The fuzzy-based rules map the input and output membership functions. The fuzzy inference engine is based on fuzzy IF-THEN-based rules, which are ultimately written by a professional designer in the related field. The rules of the fuzzy-based system hold at most $(n \times$ $m \times k)$ IF-THEN rules, where $n, m$, and $k$ are the numbers of membership functions characterized by the input variables. These memberships are connected using special fuzzy logic operators. In our case, we used the AND operator (minimum $(x, y, z)$,$) of 27$ rules when designing the fuzzy IF-THEN-based rules for our fuzzy inference engine, as shown in Table 1. For example, IF the node residual energy is high and node speed is LOW AND hop count is SHORT, THEN the node trust value is VERY HIGH. This means that this node is a trusted node (more qualified) to be a part of a stable route. In contrast, IF the node residual energy is LOW AND node speed is HIGH AND the hop count is LONG, THEN the node trust value is VERY LOW. This means that this node is not a qualified node, and it could cause established routes to fail if it is used.

Defuzzification is a mathematical method that uses a weighted mean approach to extract a crisp output value from the aggregation of the fuzzy output representation. There are different approaches used to find the crisp output. The centroid method of defuzzification is used in this proposed model. The mathematical expression for the centroid defuzzification method is as follows.

$$
\operatorname{COG}=\frac{\int \mu_{\mathrm{A}}(x) \cdot x \mathrm{~d} x}{\int \mu_{\mathrm{A}}(x) \mathrm{d} x}
$$

Here, $\mu_{\mathrm{A}}(x)$ represents the weight of the output membership function defined in Eqs. 1 and 2, $x$ denotes the centroid of each output membership function, and center of gravity (COG) denotes the crisp value of the defuzzifier output $[29,30]$.

\subsection{Description of the operation of fuzzy logic algorithm}

The description of the proposed Fuzzy logic algorithm can be summarized in four basic steps of fuzzification, IF-THEN rule evaluation, output aggregation, and defuzzification to calculate the crisp value. These steps are described as the following:

Step 1: Fuzzification of input crisp parameter values The input parameters, in our case, are node residual energy, node speed, and the number of

Table 2 Parameter values of simulation scenario

\begin{tabular}{ll}
\hline Parameters & Values \\
\hline Network simulator & NS-2.35 \\
Routing protocols & AODV, Fuzzy AODV, MBCR \\
Wireless Mac Layer protocol & IEEE 802.11 \\
Number of nodes & 50 \\
Simulation area & $900 \times 900 \mathrm{~m}$ \\
Wireless transmission range & $250 \mathrm{~m}$ \\
Mobility model & Random waypoint model \\
Pause time & $10-20-30-40-50-60-70-80-90-100 \mathrm{~s}$ \\
Simulation time & $300 \mathrm{~s}$ \\
Interface queue size & 50 \\
Size of packet & 512 bytes/packet \\
Application Layer & FTP
\end{tabular}




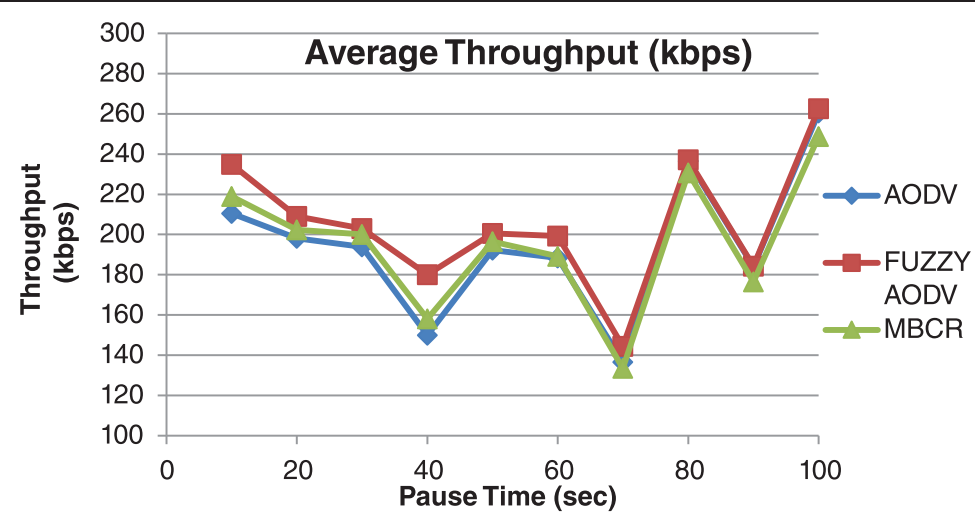

Fig. 6 Average network throughput vs. pause time

hop counts is defined by their membership functions as shown in Fig. 5. Depending on the three input crisp values, we can find the membership degree of each input by intersecting the input value with the membership function.

Step 2: Evaluation of IF-THEN rules

The membership degrees found in Step 1 are fed to IF-THEN-based rules to determine the output fuzzy set. The AND operator is used to select the minimum membership values out of the three input membership values.

Step 3: Aggregation of outputs

In this step, the system collects, in the union form, all outputs that result from applying the IF-THEN rules and then apply the OR operator to these outputs to select the maximum evaluating values to construct a new aggregate fuzzy set.

Step 4: Defuzzification process

The centroid method (center of gravity) [31] is applied to the new aggregate function obtained in Step 3 to calculate the node trust value by using Eq. 1.

\section{Simulation environment}

\subsection{Simulation parameters}

Our simulation study considers a network area of size $900 \times 900 \mathrm{~m}^{2}$ and 50 wireless mobile nodes randomly distributed across the simulation area with a maximum speed of $20 \mathrm{~m} / \mathrm{s}$. The parameter values of the performance simulation are listed in Table 2.

\subsection{Performance metrics}

Routing protocols of MANET performance can be evaluated using many quantitative metrics. We have used a popular performance-evaluated metric in our wireless ad hoc routing protocol simulation.

\subsubsection{Average network throughput}

It can be expressed as the amount of data packets successfully arrived at the final destination per unit of the simulation period time.

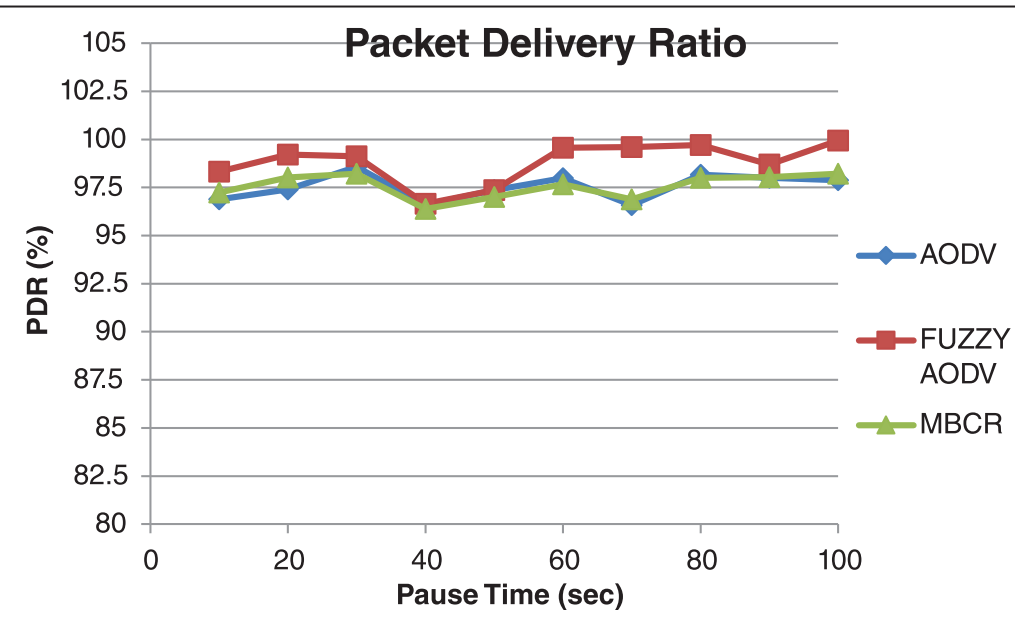

Fig. 7 PDR vs. pause time 


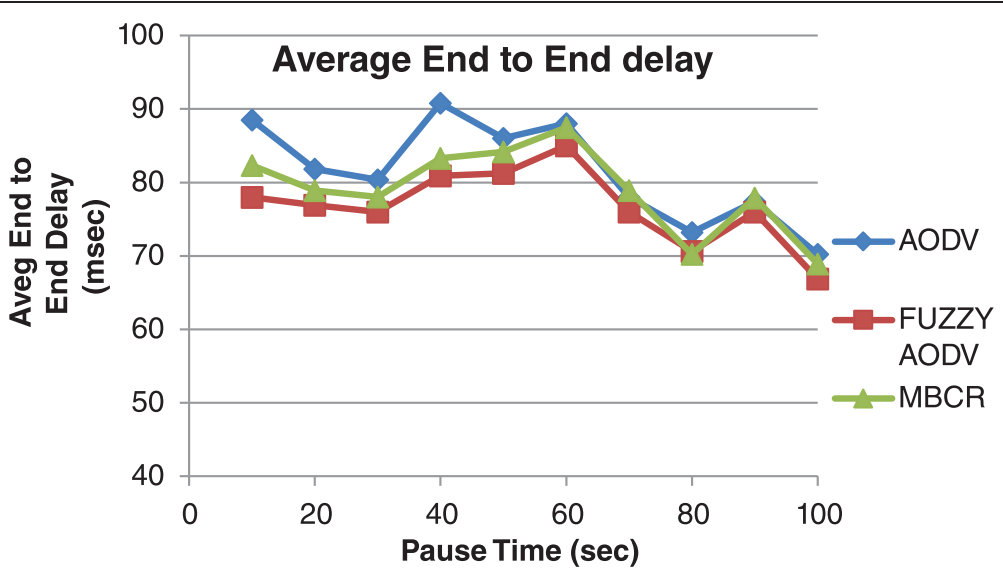

Fig. 8 Average end-to-end delay vs. pause time

\subsubsection{Packet delivery ratio (PDR)}

It can be expressed as the ratio of the packets successfully arrived at the destination nodes to the packets transmitted by the source nodes.

\subsubsection{Average routing overhead load}

It can be expressed as the total number of all overhead routing control packets sent from all nodes within the entire MANET network over the simulation time

\subsubsection{Average end-to-end delay}

It can be expressed as the average time that the data packets elapsed to transfer from the source nodes to the destination while considering all delays caused by queuing, buffering, and propagation delays.

\section{Simulation results and discussion}

Simulator version NS-2.35 [32] was used to simulate and compare the performance of the fuzzy AODV approach proposed in this work with traditional AODV and minimum battery cost routing (MBCR) for different pause times. While traditional AODV routing selects the minimum number of intermediate hops from source to destination (shortest path) route as the best route, the MBCR scheme considers the maximum values of node battery capacities as a metric for selecting the route. In MBCR routing, the minimum of the sum of the inverse remaining battery capacity (battery cost function) for all the nodes on the specific routing path is used to determine the best route, calculated, respectively, as [33]:

$$
\begin{aligned}
& R_{\mathrm{j}}=\sum_{i=0}^{D_{\mathrm{j}}-1} \frac{1}{C_{i}^{t}} \\
& R_{\mathrm{i}}=\min \left\{R_{\mathrm{j}} \mid \mathrm{j} \text { ò } \mathrm{A}\right\}
\end{aligned}
$$

where $C_{i}^{t}$ is the battery capacity and $\left(1 /{ }_{c} i^{t}\right)$ is defined as a battery cost function of node $n_{i}$ at the time $t$. As the battery capacity decreases, the battery cost function increases. Hence, the sum of battery cost $R_{\mathrm{J}}$ for route $i$ consisting of D nodes is given in Eq. 2. Equation 3 represents the minimum battery cost for route $i$, which is

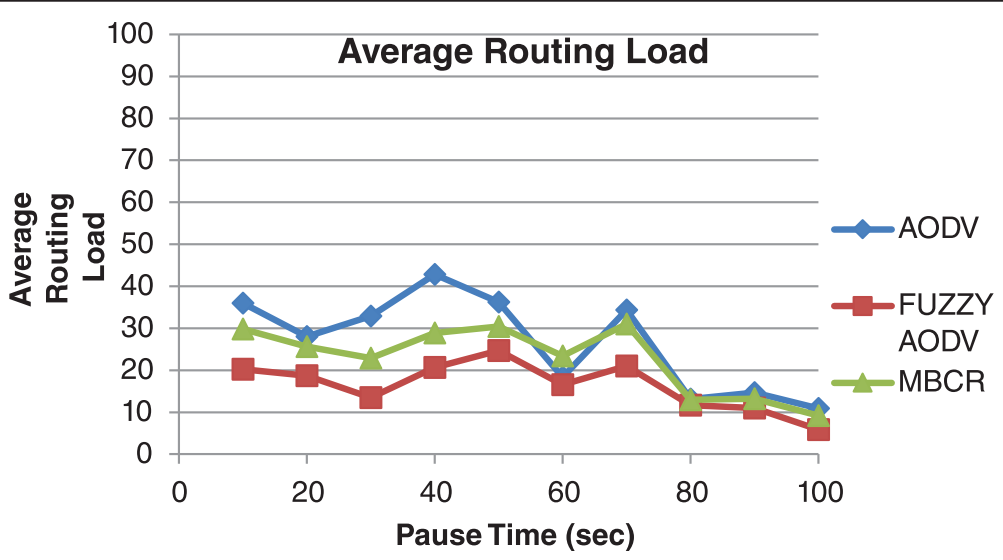

Fig. 9 Average routing loads vs. pause time 
used to find the best route with the maximum remaining battery capacity from among set A, which contains all possible routes to destinations in the MANET.

\subsection{Network throughput and packet delivery ratio}

Figure 6 shows the average network throughput of the fuzzy AODV routing protocol compared with the classical AODV and MBCR routing protocols. It is clear that the number of packets received by the destination nodes of the modified fuzzy AODV protocol was slightly higher than that received by the classical AODV and MBCR during periods of high node mobility (low pause times). This is caused by the random waypoint mobility model used in our simulation scenario, where each node moves independently and reaches a specific position in the terrain for one simulation iteration and stays in that position for a limited period of time (pause time). It then chooses a new direction and speed for the next iteration, and so on, until the overall simulation time is over. Hence, in low pause times (high-mobility scenarios), the routes established in the AODV and MBCR routing scenarios are broken more frequently than with fuzzy AODV. This leads to a reduction in the number of packets that successfully reach the destination node over the total simulation time in singlemetric simple routing protocols when compared with the more stable routes established by the fuzzy AODV protocol. Figure 7 shows the packet delivery ratio for the classical AODV, MBCR, and fuzzy AODV protocols when the node pause time varies. It is clear that fuzzy AODV performs better than the other two routing protocols and achieves a higher packet delivery ratio (PDR) than the traditional AODV and MBCR routing protocols. These protocols show no significant difference in the PDR values for different pause time scenarios.

\subsection{Average end-to-end delay}

As shown in Fig. 8, fuzzy AODV has a lower average delay than classic AODV and MBCR for pause time values of less than $60 \mathrm{~s}$. This is due to the fact that more frequent route breaks occur in the high-mobility scenarios of simple single-metric routing protocols compared with that of the modified fuzzy AODV protocol, which increases the packet delays reaching the destination nodes in a MANET. In addition, it shows that packet delay decreases gradually with increasing pause times of more than $60 \mathrm{~s}$. Generally, the average delay using fuzzy AODV gives a better end-to-end delay performance than traditional AODV and MBCR.

\subsection{Average routing control overhead load}

Figure 9 shows the effects of node mobility on routing control overhead packets in the three routing protocol scenarios used in this study. It is clear that the number of route overhead packets decreases as the node pause time increases. Furthermore, traditional AODV has the highest average number of control overhead packets compared with fuzzy AODV and MBCR routing protocols, where the fuzzy AODV protocol minimizes the number of control overhead packets broadcast over the MANET network, as discussed in the previous sections.

Hence, the advantages of the fuzzy AODV protocol lie in enhancing the data transmission continuity in network throughput and PDR terms as well as decreasing the amount of control overhead load of the MANETs.

\section{Conclusions}

In this study, we introduced a fuzzy logic scheme to improve MANET performance. Fuzzy logic appears to be an efficient approach for constructing robust routes and avoiding some of the shortcomings of simple singlemetric routing protocols such as the traditional AODV and $M B C R$ reactive routing protocols. The fuzzy logic AODV scheme applied in this work has adaptive properties and better performance than the original AODV routing protocol. The simulation results show that, by increasing node mobility, the control overhead packets and the end-to-end delays are better than those of the traditional AODV and MBCR protocols. Although the network throughput is slightly increased, it is still at an acceptable level. Additionally, the proposed fuzzy logic AODV algorithm performs better in high-mobility environments. In the future, more factors and metrics may be considered in the fuzzy inference engine to enhance the route selection decision-making.

\section{Competing interests}

The authors declare that they have no competing interests.

\section{Authors' information}

Dr. Emre Ozen contributed effectively in writing $\mathrm{C}++$ codes network simulator NS-2 for our manuscript published paper.

\section{Author details}

${ }^{1}$ Department of Computer Engineering, Eastern Mediterranean University, Famagusta, N. Cyprus. ${ }^{2}$ School of Computing and Technology, Eastern Mediterranean University, Famagusta, N. Cyprus.

Received: 11 August 2015 Accepted: 16 October 2015 Published online: 28 October 2015

\section{References}

1. F Legendre, T Hossmann, F Sutton, B Plattner, 30 years of wireless ad hoc networking research: what about humanitarian and disaster relief solutions? What are we still missing? ACWR '11 (ACM, Amritapuri, 2011)

2. S Verma, P Nayak, R Agarwa, Energy efficient routing in mobile adhoc networks based on AODV protocol. Int J Comp Sci Issues 9(6), 344 (2012)

3. O Younis, S Fahmy, Constraint-based routing in the internet basic principles and recent research. IEEE CFIMSOC Surveys Tumrial (2003). doi:10.1109/ COMST.2003.5342226

4. P Li, S Guo, S Yu, AV Vasilakos, Reliable multicast with pipelined network coding using opportunistic feeding and routing. IEEE Trans Parallel Distri Sys 25(12), 3264-3273 (2014)

5. W Pedrycz, A Vasilakos, S Karnouskos, Special issue on computational intelligence in telecommunications networks and internet services - part II. IEEE Trans Sys Man Cybernetics_Part C: App Rev (2003). doi:10.1109/ TSMCC.2003.819319 
6. M Abolhasan, T Wysocki, E Dutkiewicz, A review of routing protocols for mobile ad hoc networks. Ad Hoc Netw 2(1), 1-22 (2004)

7. VP Patil, Reactive and proactive routing protocol performance evaluation for qualitative and quantitative analysis in mobile ad hoc network. Int I Sci Res Pub 2(9), 1-8 (2012)

8. TH Clausen, P Jacquet, L Viennot, Comparative study of routing protocols for mobile ad-hoc NETworks (INRIA Rocquencourt, Projet Hipercom, Domaine de Voluceau, 2002)

9. A Abd Rahman, ZA Zukarnain, Performance comparison of AODV, DSDV and I-DSDV routing protocols in mobile ad hoc networks. Eur J Sci Res 31(4), 566-576 (2009)

10. T Meng, F Wu, Z Yang, G Chen, AV Vasilakos, Spatial reusability-aware routing in multi-Hop wireless networks. IEEE Trans Comput (2015). doi:10.1109/ TC.2015.2417543

11. XM Zhang, Y Zhang, F Yan, AV Vasilakos, Interference-based topology control algorithm for delay-constrained mobile ad hoc networks. IEEE Trans Mobile Comp (2015). doi:10.1109/TMC.2014.2331966

12. S Marwaha, D Srinivasan, CK Tham, A Vasilakos, Evolutionary fuzzy multiobjective routing for wireless mobile Ad Hoc networks. Evolutionary Computation, CEC2004 Congress 2, 1964-1971 (2004)

13. S Srivastava, AK Daniel, An efficient routing protocol under noisy environment for mobile ad hoc networks using fuzzy logic. Int J Adv Res Artif Intell (2013). doi:10.14569/IJARAI.2013.020901

14. GS Hundal, SK Gupta, R Bedi, Adaptive approach to find a stable path between nodes in MANET. Int J Curr Eng Technol 4(4), 2898-2901 (2014)

15. $B X u, Y L i, A$ novel link stability and energy aware routing with tradeoff strategy in mobile ad hoc networks. J Commun 9(9), 706-713 (2014)

16. Z Zhang, Z Li, J Chen, An enhanced AODV route repairing mechanism in wireless ad-hoc sensor network. J Commun 9(8), 651-657 (2014)

17. M Ghorbani, AM Saghiri, MR Meybodi, A novel adaptive version of AODV routing protocol based on learning automata utilizing cognitive networks concept. Tech J Eng App Sci 3(22), 3064-3070 (2013)

18. SH Nasiri, M Fathy, EZ Khosrafi, A Shojaeifard, Improving link reliability in mobile ad hoc networks using fuzzy nodes. Communications \& information technology marathon beach, Attica, Greece, 2008, pp. 1-3

19. G Lim, K Shin, S Lee, H Yoon, J Ma, Link stability and route lifetime in ad-hoc wireless networks. Proc Int Conf Paral Proc Workshops (2002). doi:10.1109/ ICPPW.2002.1039720

20. S Das, Ad hoc on-demand distance vector (AODV) routing (Santa Barbara, Network Working Group, Nokia Research Center, University of California, 2003)

21. SR Das, CE Perkins, EM Roycr, Performance comparison of Two on-demand routing protocols for ad hoc networks. IEEE Infocom (2000). doi:10.1109/ INFCOM.2000.832168

22. LA Zadeh, Fuzzy sets. Inf Control 8, 338-353 (1965)

23. X Ban, XZ Gao, X Huang, H Yin, Stability analysis of the simplest takagi-sugeno fuzzy control system using circle criterion (IEEE International Conference on Fuzzy Systems, Vancouver, 2006)

24. A Vasilakos, MP Saltouros, AF Atlassis, W Pedrycz, Optimizing QoS routing in hierarchical ATM networks using computational intelligence techniques. IEEE Trans Sys Man Cyber_-Part c: App Rev 33(3), 297-312 (2003)

25. A Vasilakos, C Ricudis, K Anagnostakis, W Pedrycz, A Pitsillides, Evolutionaryfuzzy prediction for strategic QoS routing in broadband networks. Fuzzy systems proceedings, IEEE world congress on computational intelligence. IEEE Int Conf 2, 1488-1493 (1998)

26. F Dernoncourt, Introduction to fuzzy logic (MIT, Massachusetts, 2013)

27. W El-Hajj, D Kountanis, A Al-Fuqaha, M Guizani, A fuzzy-based hierarchical energy efficient routing protocol for large scale mobile ad hoc networks (FEER). IEEE Commun Soc (2006). doi:10.1109/ICC.2006.255628

28. M Youssef, M Ibrahim, M Abdelatif, L Chen, AV Vasilakos, Routing metrics of cognitive radio networks: a survey. IEEE Commun Soc Tutor 16(1), 92-109 (2014)

29. GW Nurcahyo, SM Shamsuddin, RA Alias, MN Sap, Selection of defuzzification method to obtain crisp value for representing uncertain data in a modified sweep algorithm. JCT 3(3), 22-28 (2003)

30. G Kalpana, M Punithavalli, Fuzzy logic technique for gossip based reliable broadcasting in mobile ad hoc networks. J Theoretical App Info Tech 51(3), 1992-8645 (2013)

31. R Saneifard, R Saneifard, A method for defuzzification based on centroid point. Turkish J Fuzzy Sys 2(1), 36-44 (2011)

32. Network Simulator, NS-2, http://www.isi.edu/nsnam/-ns/.

33. C-K Toh, Maximum battery life routing to support ubiquitous mobile computing in wireless ad hoc networks. IEEE Commun Mag 39, 138-147 (2001)

\section{Submit your manuscript to a SpringerOpen ${ }^{\odot}$ journal and benefit from:}

- Convenient online submission

$\checkmark$ Rigorous peer review

- Immediate publication on acceptance

- Open access: articles freely available online

- High visibility within the field

- Retaining the copyright to your article

Submit your next manuscript at springeropen.com 